\title{
Generalized Statistics Framework for Rate Distortion Theory
}

\author{
R. C. Venkatesan \\ Systems Research Corporation \\ Aundh, Pune 411007, India
}

\begin{abstract}
A variational principle for the rate distortion (RD) theory is formulated within the ambit of the generalized nonextensive statistics of Tsallis. Alternating minimization schemes for the nonextensive RD theory are derived. The advantages of employing nonextensive statistics vis-á-vis Boltzmann-Gibbs statistics in data compression are exemplified with the aid of numerical simulations. The theory of the Information Bottleneck method is established within a generalized statistics framework.
\end{abstract}

\section{INTRODUCTION}

The generalized (nonextensive) statistics of Tsallis [1,2] has recently been the focus of much attention in statistical physics, and allied disciplines. Nonextensive statistics has found applications in a wide spectrum of disciplines ranging from condensed matter physics to financial mathematics. A continually updated bibliography of works related to nonextensive statistics may be found at http://tsallis.cat.cbpf.br/biblio.htm.

Nonextensive statistics generalizes the extensive Boltzmann-Gibbs statistics, and has much utility in complex systems. Some of features possessed by complex systems, which invite the use of nonextensive statistics, are long range correlations, fluctuations, ergodicity, chirality and fractal behavior, amongst others. By definition, the Tsallis entropy is defined in terms of discrete variables as

$$
S_{q}(x)=\frac{1-\sum_{x} p^{q}(x)}{1-q}, \text { where, } \sum_{x} p(x)=1 .
$$

The constant $q$ is referred to as the nonextensivity parameter. Given two independent variables $x$ and $y$, one of the fundamental consequences of nonextensivity is demonstrated by the pseudo-additivity relation

$$
S_{q}(x y)=S_{q}(x)+S_{q}(y)+(1-q) S_{q}(x) S_{q}(y) .
$$

Here, (1) and (2) imply that extensive statistics is recovered as $q \rightarrow 1$. Taking the limit $q \rightarrow 1$ in (1) and evoking l'Hospital's rule, $S_{q}(x) \rightarrow S(x)$, the Shannon entropy.

The generalized Kullback-Leibler divergence (K-Ld) is of the form [3]

$$
I_{q}(p(x) \| r(x))=\sum_{x} p(x) \frac{\left(\frac{p(x)}{r(x)}\right)^{q-1}-1}{q-1} .
$$

In the limit $q \rightarrow 1$, the extensive $\mathrm{K}-\mathrm{Ld}$ is readily recovered. Akin to the Tsallis entropy, the generalized K-Ld obeys the pseudo-additivity relation [3].

Phone: +91-20-25883952, email: ravi@ systemsresearchcorp.com 
Rate distortion (RD) theory constitutes one of the cornerstones of contemporary information theory $[4,5]$. RD theory has found applications in diverse disciplines, which include data compression and clustering. Deterministic annealing (DA) $[6,7]$ and the information bottleneck (IB) method [8] are two influential paradigms in machine learning, that are closely related to RD theory. The representation of RD theory in the form of a variational principle, expressed within the framework of the Shannon information theory, has been established (see Chapter 13 of [4]).

The seminal paper on source coding within the framework of nonextensive statistics by Landsberg and Vedral [9], has provided the impetus for a number of investigations into the use of nonextensive information theory within the context of coding problems. The works of Yamano $[10,11]$ represent a sample of some of the prominent efforts in this regard.

The primary objective of this paper is to re-formulate RD theory within the ambit of nonextensive statistics. Further, alternating minimization numerical algorithms based on the Blahut-Arimoto scheme [12], are derived for the nonextensive RD theory. Section IV highlights the distinctions between extensive vis-á-vis nonextensive statistics with the aid of numerical simulations. Therein, it is established that nonextensive RD models possess a lower threshold for the minimum compression information in the distortion-compression plane, as compared to equivalent RD models derived on the basis of the Boltzmann-GibbsShannon framework.

This observation demonstrates the qualitative advantages of nonextensive RD models to perform data compression vis-á-vis extensive RD models. Section V concludes this paper by re-formulating the theoretical analysis of the IB method [8], within a generalized statistics framework. One of the noteworthy results of the IB method is the self-consistent derivation of the distortion measure from the joint statistics of the source distribution $X$ and the relevance variable $Y$ [8]. Section $\mathrm{V}$ tacitly demonstrates that this result carries over into the nonextensive regime.

\section{Select Theory in Generalized Statistics}

\section{A. Selected results from q-algebra}

Generalized statistical mechanics often utilizes results from q-algebra [13], in order to derive equations that resemble their counterparts obtained from Boltzmann-Gibbs statistics. Herein, selected results utilized in this paper are described. The Tsallis entropy (1), and, the generalized K-Ld (3) may be written as

$$
S_{q}(p(x))=-\sum_{x} p(x)^{q} \ln _{q} p(x)
$$

and,

$$
I_{q}(p(x) \| r(x))=-\sum_{x} p(x)^{q} \ln _{q} \frac{r(x)}{p(x)},
$$

respectively. Here, the $q$-deformed logarithm and the $q$-deformed exponential are

$$
\ln _{q}(x)=\frac{x^{1-q}-1}{1-q}, \text { and }, e_{q}^{x}=\left\{\begin{array}{l}
{[1+(1-q) x]^{1 /(1-q)} ; 1+(1-q) x \geq 0} \\
0 ; \text { otherwise, }
\end{array}\right.
$$


respectively. In this paper, $0<q<1$ in order to preserve the jointly convex nature of the generalized K-Ld. A value $q>1$ yields a negative channel capacity [11], which is undesirable. Some of the salient results of $q$-algebra, that are employed in this paper are

$$
\begin{aligned}
& \ln _{q}(x y)=\ln _{q}(x) \oplus_{q} \ln _{q}(x)=\ln _{q}(x)+\ln _{q}(y)+(1-q) \ln _{q}(x) \ln _{q}(y), \\
& \ln _{q}(x / y)=\ln _{q}(x) \ominus_{q} \ln _{q}(x), \text { where, } \ominus_{q} y=\frac{-y}{1+(1-q) y)}
\end{aligned}
$$

Note that the results of $q$-algebra are not constructions specifically manufactured to express results obtained from generalized statistical mechanics, in a form analogous to their counterparts in extensive statistics. The results of $q$-algebra have a long lineage, and the existence of certain results (specifically the $q$-deformed exponential) were known to Leibniz [13].

\section{B. Constraints}

Generalized statistics has utilized a number of constraints to define expectations. The original Tsallis (OT) constraints of the form $\langle A\rangle=\sum_{i} p_{i} A_{i}$ [1], were convenient owing to their similarity to the maximum entropy constraints. These were abandoned because of difficulties encountered in obtaining an acceptable form for the partition function. These were subsequently replaced by the Curado-Tsallis (C-T) [14] constraints $\langle A\rangle_{q}=\sum_{i} p_{i}^{q} A_{i}$, . The C-T constraints were later replaced by the normalized Tsallis-Mendes-Plastino (T-MP) constraints [15] $\langle A\rangle_{q}=\sum_{i} \frac{p_{i}^{q}}{\aleph_{q}(x)} A_{i} ; \aleph_{q}(x)=\sum_{i} p_{i}^{q}$. The dependence of the expectation value on the normalized $p d f \aleph_{q}(x)$, rendered the T-M-P constraints to be self-referential.

A recent work [16] has described a methodology to "rescue" the OT constraints, and, has linked the OT, C-T, and, T-M-P constraints. The present paper utilizes the OT constraints, by deriving a procedure analogous to that in [16]. This enables the nonextensive variational principles for the RD theory and the IB method to be cast in a manner that parallels the extensive case.

\section{Nonextensive Rate Distortion Variational PRinciple}

Let $X \in \Xi$ be discrete random variable, distributed according to the marginal $p d f p(x)$. Let $\tilde{X} \in \tilde{\Xi}$ be another discrete random variable, distributed according to the marginal $p d f$ $p(\tilde{x})$. Here, $\tilde{X}$ is a compressed representation (quantized codebook) of $X$, defined through a (possibly stochastic) mapping between each value $x \in \Xi$ to a representative value $\tilde{x} \in \tilde{\Xi}$. This mapping is characterized by the conditional probability $p(\tilde{x} \mid x)$, which induces a soft partitioning (assignment) of the $X$ discrete random variables. Each $x \in \Xi$ relates to all $\tilde{x} \in \tilde{\Xi}$ through a normalized conditional $p d f$ conditional probability $p(\tilde{x} \mid x)$.

Consider the nonextensive RD Lagrangian [4]

$$
L_{R D}^{q}[\tilde{x} \mid x]=I_{q}(X ; \tilde{X})+\beta\langle d(x, \tilde{x})\rangle_{p(x, \tilde{x})},
$$

subject to the normalization of the conditional probability $\sum_{x, \tilde{x}} p(x \mid \tilde{x})$. The Lagrange multiplier $\beta$ is hereafter referred to as the inverse temperature $[6,7]$. The distortion measure is denoted by $d(x, \tilde{x})$, which is taken to be the Euclidean square distance for most problems in science and engineering [7]. Here, $I_{q}$ is the generalized K-Ld defined by 


$$
I_{q}(X ; \tilde{X})=-\sum_{x, \tilde{x}} p(x, \tilde{x}) \ln _{q}\left(\frac{p(x) p(\tilde{x})}{p(x) p(\tilde{x} \mid x)}\right)=\sum_{x, \tilde{x}} \frac{p(x) p(\tilde{x} \mid x)\left(\frac{p(\tilde{x} \mid x)}{p(\tilde{x})}\right)^{q-1}-1}{q-1}
$$

Here, the $q$-deformed logarithm is $\ln _{q}(x)$. Defining $R_{q}(D)=\min _{p(\tilde{x} \mid x):\langle d(x, \tilde{x}) \leq D\rangle} I_{q}(X ; \tilde{X})$,

$$
\delta R_{q}(D)=\delta I_{q}(X ; \tilde{X})+\beta \delta\langle d(x, \tilde{x})\rangle_{p(x, \tilde{x})}=0 \Rightarrow \frac{\delta I_{q}(X ; \tilde{X})}{\delta\langle d(x, \tilde{x})\rangle_{p(x, \tilde{x})}}=-\beta .
$$

The joint $p d f p(x, \tilde{x})$ is taken to be normalized, thus, $\sum_{x, \tilde{x}} p(x, \tilde{x})=1$. Expanding $p(x, \tilde{x})=$ $p(x) p(\tilde{x} \mid x),(10)$ acquires the form

$$
L_{R D}^{q}[\tilde{x} \mid x]=-\sum_{x, \tilde{x}} \frac{p(x) p(\tilde{x} \mid x)\left(\frac{p(\tilde{x} \mid x)}{p(\tilde{x})}\right)^{q-1}-1}{(1-q)}+\sum_{x, \tilde{x}} \beta d(x, \tilde{x}) p(x) p(\tilde{x} \mid x)+\sum_{x, \tilde{x}} \lambda(x) p(\tilde{x} \mid x) .
$$

Defining $p(\tilde{x})=\sum_{x} p(x) p(\tilde{x} \mid x)$, and noting that $\frac{\delta p(\tilde{x})}{\delta p(\tilde{x} \mid x)}=p(x)$, the variational derivative of (11) yields

$$
\frac{\delta L_{R D}^{q}[\tilde{x} \mid x]}{\delta p(\tilde{x} \mid x)}=p(x)\left[\frac{q}{q-1}\left(\frac{p(\tilde{x} \mid x)}{p(\tilde{x})}\right)^{q-1}+\beta d(x, \tilde{x})+\tilde{\lambda}(x)\right]=0 .
$$

It is important to note that conditional probabilities first acquired prominence in nonextensive statistics owing to their utility in characterizing quantum entanglement. This was accomplished in the seminal work of Abe and Rajagopal [17]. In (12) the scaled Lagrange multiplier $\tilde{\lambda}(x)=\frac{\lambda(x)}{p(x)}-p(x)^{(1-q)}$, evaluated for each value of $x$, enforces the normalization of the conditional probability. Note that the normalization Lagrange multiplier acts as a "reservoir" into which terms independent of $\tilde{x}$ may be absorbed. This feature is also utilized when deriving the theoretical framework for the nonextensive IB method in Section $V$. Expanding (12), yields

$$
p(\tilde{x} \mid x)=p(\tilde{x})\left[\frac{(1-q)}{q}\{\tilde{\lambda}(x)+\beta d(x, \tilde{x})\}\right]^{1 /(q-1)} .
$$

The scaled normalization Lagrange multiplier is obtained as follows. Multiplying the terms in the square brackets in (12) by the conditional probability $p(\tilde{x} \mid x)$, and summing over $\tilde{x}$ yields

$$
\frac{q}{q-1} \sum_{\tilde{x}} p(\tilde{x})\left(\frac{p(\tilde{x} \mid x)}{p(\tilde{x})}\right)^{q}+\underbrace{\beta \sum_{\tilde{x}} d(x, \tilde{x}) p(\tilde{x} \mid x)}_{\beta\langle d(x, \tilde{x})\rangle_{p(\tilde{x} \mid X=x)}}+\tilde{\lambda}(x) \sum_{\tilde{x}} p(\tilde{x} \mid x)=0 .
$$

Evoking the normalization condition for the conditional $p d f$ with the source sample fixed at $X=x$, i.e. $\sum_{\tilde{x}} p(\tilde{x} \mid X=x)=1$, yields

$$
\tilde{\lambda}(x)=\frac{q}{(1-q)} \aleph_{q}(x)-\beta\langle d(x, \tilde{x})\rangle_{p(\tilde{X} \mid X=x)} .
$$


Here, $\aleph_{q}(x)=\sum_{\tilde{x}} p(\tilde{x})\left(\frac{p(\tilde{x} \mid x)}{p(\tilde{x})}\right)^{q}$. Expectations with respect to $p(\tilde{X} \mid X=x)$ are often encountered in random encoding strategies.

The conditional $p d f p(\tilde{x} \mid x)$ acquires the form

$$
p(\tilde{x} \mid x)=p(\tilde{x})\left\{\aleph_{q}(x)+\frac{(1-q)}{q} \beta \Delta d(x, \tilde{x})\right\}^{1 /(q-1)},
$$

where, $\Delta d(x, \tilde{x})=\left\{d(x, \tilde{x})-\langle d(x, \tilde{x})\rangle_{p(\tilde{X} \mid X=x)}\right\}$. Re-arranging the terms in (16) yields

$$
p(\tilde{x} \mid x)=\frac{p(\tilde{x})\left\{1-\frac{(q-1) \beta d(x, \tilde{x})}{q \aleph_{q}(x)+(q-1) \beta\langle d(x, \tilde{x})\rangle_{p(\tilde{X} \mid X=x)}}\right\}^{1 /(q-1)}}{\left[q \aleph_{q}(x)+(q-1) \beta\langle d(x, \tilde{x})\rangle_{p(\tilde{X} \mid X=x)}\right]^{1 /(1-q)}}=\frac{p(\tilde{x})\left\{1-(q-1) \beta^{*} d(x, \tilde{x})\right\}^{1 /(q-1)}}{\Im_{R D}^{1 /(1-q)}},
$$

where the effective inverse temperature $\beta^{*}=\frac{\beta}{q \aleph_{q}(x)+(q-1) \beta\langle d(x, \tilde{x})\rangle_{p(\tilde{X} \mid X=x)}}$. Transforming $q \rightarrow 2-q^{*}$ in the numerator and evoking (6), (17) is expressed in the form of a $q$-deformed exponential

$$
p(\tilde{x} \mid x)=\frac{p(\tilde{x}) \exp _{q^{*}}\left(-\beta^{*} d(x, \tilde{x})\right)}{\tilde{Z}\left(x, \beta^{*}\right)} .
$$

The term $1-\left(1-q^{*}\right) \beta^{*} d(x, \tilde{x})$ in the numerator of (17) is a manifestation of the Tsallis cutoff condition [16]. This implies that solutions of (18) are valid when $\beta^{*} d(x, \tilde{x})<1 /\left(1-q^{*}\right)$. Note that $\Im_{R D}^{1 /(1-q)}=\tilde{Z}\left(x, \beta^{*}\right)$, the partition function evaluated at each value of the source distribution. To provide the nonextensive RD theory with a statistical physics connotation [5-7], the effective nonextensive RD Helmholtz free energy is

$$
F_{R D}^{q}\left(\beta^{*}\right)=\frac{-1}{\beta^{*}}\left\langle\ln _{q} \tilde{Z}\left(x, \beta^{*}\right)\right\rangle_{p(x)}=\frac{1}{\beta^{*}}\left\langle\frac{\Im_{R D}-1}{q-1}\right\rangle_{p(x) .}
$$

Solution of (18) may be viewed from two distinct perspectives, i.e. the canonical perspective and the parametric perspective. Owing to the self-referential nature of the effective inverse temperature $\beta^{*}$, the analysis and solution of (18) within the context of the canonical perspective is a formidable undertaking. This will be studied in an immediately forthcoming publication. For practical applications, the parametric perspective is utilized by evaluating the conditional $p d f p(\tilde{x} \mid x)$, employing the nonextensive Blahut-Arimoto algorithm, for a-priori specified $\beta^{*} \in[0, \infty]$. Note that within the context of the parametric perspective, the self-referential nature of $\beta^{*}$ vanishes. The inverse temperature $\beta$ and the effective inverse temperature $\beta^{*}$ relate as

$$
\beta=\frac{q \aleph_{q}(x) \beta^{*}}{\left[1-\beta^{*}(q-1)\langle d(x, \tilde{x})\rangle_{p(\tilde{X} \mid X=x)}\right]} .
$$

As will be presented in an immediately forthcoming paper, information theoretic and thermodynamic quantities in "canonical space" ( $\beta$ dependent) relate to their counterparts in "parametric space" ( $\beta^{*}$ dependent) through a Legendre transform-like structure. The Blahut-Arimoto algorithm for nonextensive RD theory is described in Algorithm 1. 


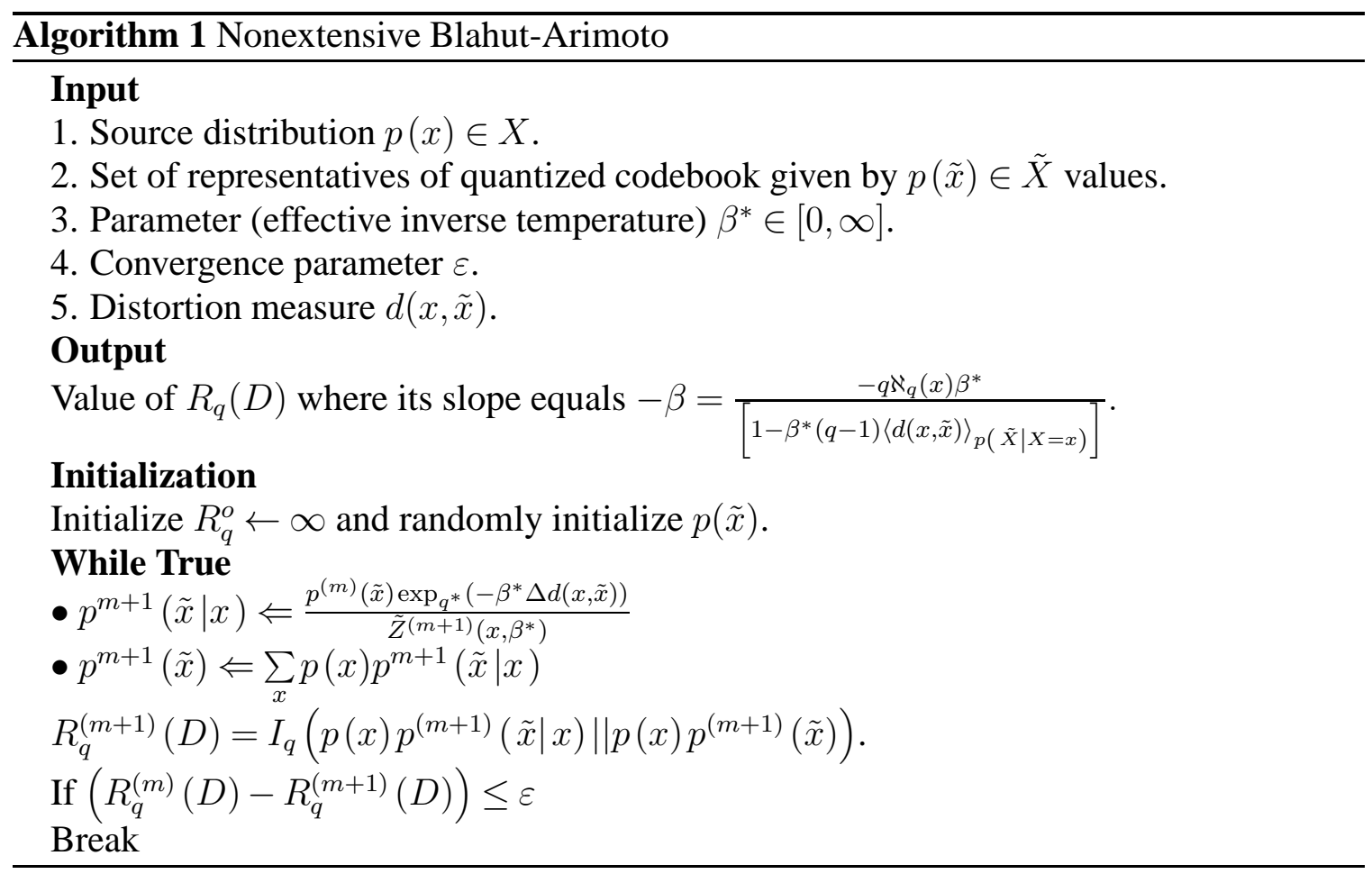

\section{Numerical Simulations AND PhysicAl InTERPREtations}

The qualitative distinctions between nonextensive statistics and extensive statistics is demonstrated with the aid of the respective RD models. To this end, a sample of 1000 two-dimensional data points is drawn from three spherical Gaussian distributions with centers $(2,3.5),(0,0),(0,2)$ (the quantized codebook). The priors and standard deviations are $0.3,0.4,0.3$, and, $0.2,0.5,1.0$, respectively.

Fig. 1 depicts the extensive and nonextensive RD curves, with the constituent discrete points overlaid upon them. Each curve has been generated for 1000 values of $\beta \in[.1,2.5]$, and $\beta^{*} \in[.1,100]$, respectively. It is observed that the nonextensive theory for the nonextensivity parameter $q$ in the range $0<q<1$ uniformly exhibit a lower threshold for the minimum achievable compression-information in the distortion-compression plane, as compared to the extensive case. Note that for the nonextensive cases, the slope of the tangent drawn at any point on the RD curve is the negative of the inverse temperature $-\beta$ and not $-\beta^{*}$. At the commencement, $\beta \rightarrow 0, \beta^{*} \rightarrow 0$, the Blahut-Arimoto algorithm solves for the compression phase. As $\beta$ and $\beta^{*}$ increase, the data points undergo soft clustering around the cluster centers. The hard clustering regime signifies regions where $\beta \rightarrow \infty, \beta^{*} \rightarrow \infty$. As observed in Fig. 1, the hard clustering regions correspond to portions of the RD curves where the discrete points are tightly packed. It is observed that the nonextensive RD models undergo compression and clustering more rapidly than the equivalent extensive RD model. A primary cause for such behavior is described in Fig. 2, where the nonextensive effective inverse temperatures $\beta^{*}$ are depicted versus the corresponding inverse temperatures $\beta$, with the aid of (20). As is noticed, $\beta^{*}$ increases rapidly with marginal increases in $\beta$. Fig. 2 also describes the "evolution" of the the effective RD Helmholtz free energy with respect to $\beta^{*}$, with the aid of (19).

The above arguments result in an observation of particular significance in Fig. 1. Specif- 
ically, even for less relaxed distortion constraints $\langle d(x, \tilde{x})\rangle_{p(x, \tilde{x})}$, any nonextensive case for $0<q<1$ possesses a lower minimum compression information than the corresponding extensive case. The threshold for the minimum achievable compression-information decreases as $q \rightarrow 0$. Note that all nonextensive $R D$ curves inhabit the non-achievable region for the extensive case. Further, nonextensive RD models possessing a lower nonextensivity parameter $q$ inhabit the non-achievable regions of nonextensive RD models possessing a higher value of $q$. These features exemplify the superiority of nonextensive models to perform data compression vis-á-vis any comparable model derived from Boltzmann-Gibbs statistics.

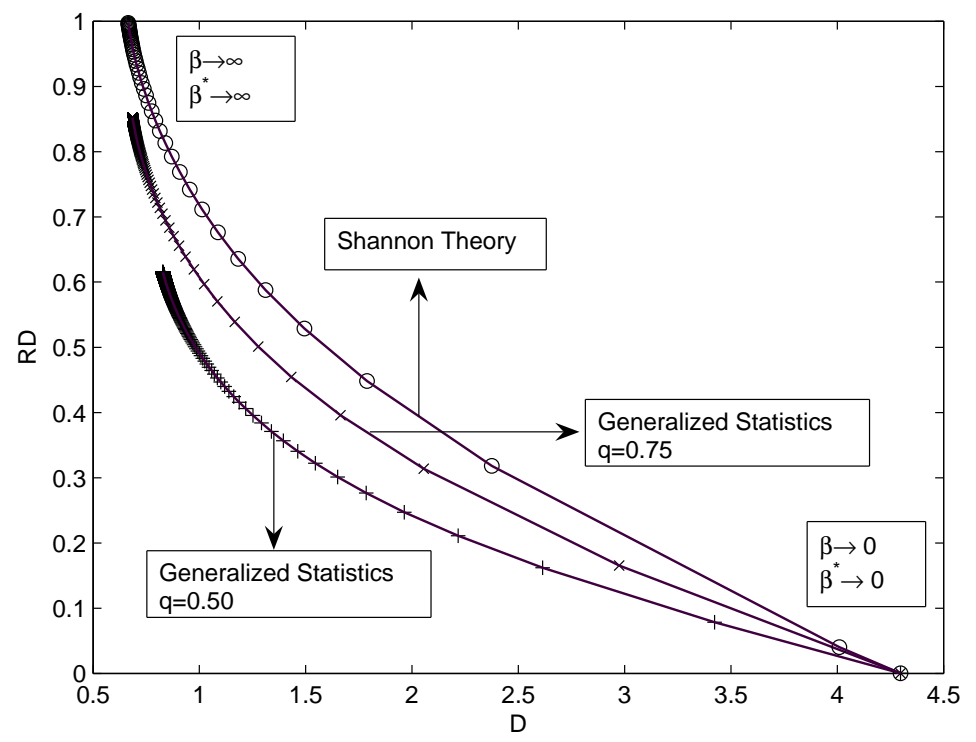

Fig. 1. Rate distortion curves for nonextensive and extensive statistics
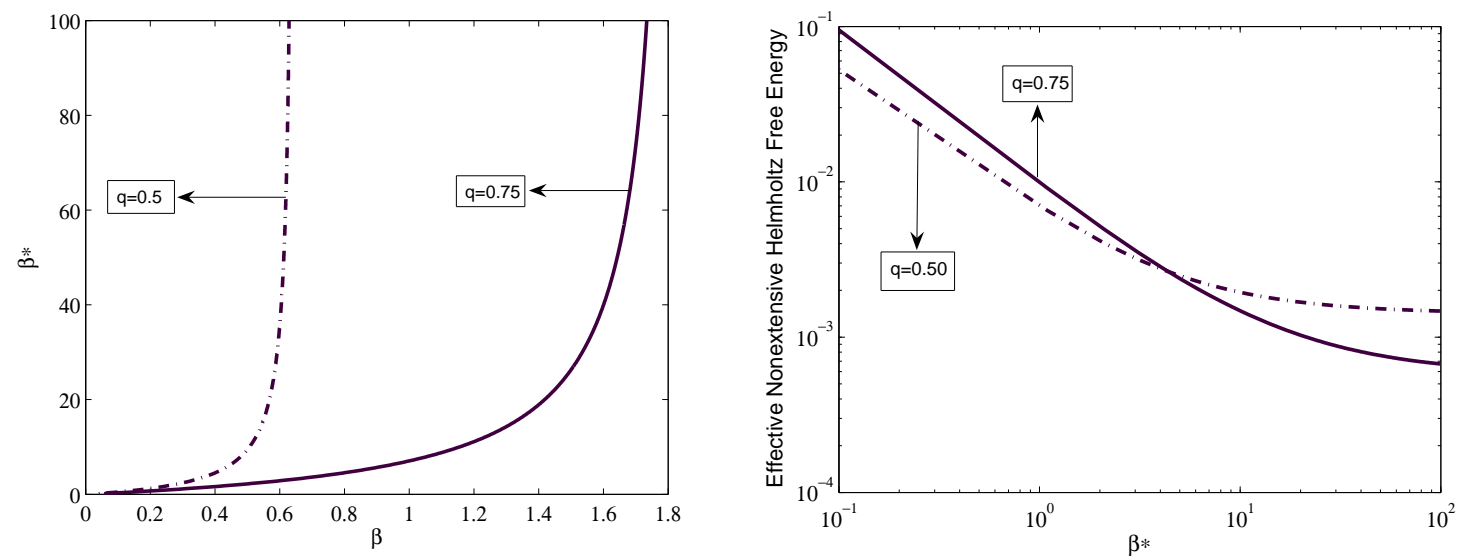

Fig. 2. Left: Curves for inverse temperature $\beta \mathrm{v} / \mathrm{s}$ effective inverse temperature $\beta^{*}$. Right: Curves for effective RD Helmholtz free energy as a function of $\beta^{*}$. 


\section{ONGOING WORK}

The analysis presented in this paper is being extended in three directions. First, nonextensive vector quantization models for image data are being developed. Next, the present work is extended to the case of the C-T and T-M-P constraints (see Section II.B.). While the information theoretic and thermodynamic quantities differ in terms of their values, preliminary simulations reveal that the overall trends and observations summarized in Section IV for the OT constraints are in accord with results obtained using the C-T and T-M-P constraints. Finally, a framework for a nonextensive IB method has been developed. The IB method [8] generalizes RD theory by introducing a principle for extracting relevant structure from data. This is accomplished by modeling structure extraction as data compression, followed by a quantification of the information preserved by the extracted structure with regards to a specific relevance variable. Here, $X \in \Xi$ is a discrete random variable, distributed according to the marginal $p d f p(x)$ - the source distribution. Further, $\tilde{X} \in \tilde{\Xi}$ is another discrete random variable, distributed according to the marginal $p d f p(\tilde{x})$. Here, $\tilde{X}$ is the bottleneck representation. The relevance variable is represented by the discrete random variables $Y \in \Phi$. The IB method is described by the Markov relation $\tilde{X} \leftrightarrow X \leftrightarrow Y$.

The nonextensive IB Lagrangian is

$$
L_{I B}^{q}[\tilde{x} \mid x]=I_{q}(X ; \tilde{X})-\beta I_{q}(\tilde{X} ; Y)-\sum_{x, \tilde{x}} \lambda(x) p(\tilde{x} \mid x) .
$$

Minimizing (21) with respect to the conditional probability $p(\tilde{x} \mid x)$ for each $x$ and $\tilde{x}$, yields

$$
\frac{q p(x)}{q-1}\left(\frac{p(\tilde{x} \mid x)}{p(\tilde{x})}\right)^{q-1}-\beta\left\{\frac{q}{q-1} \sum_{y} p(y) p^{q}(x \mid y)\left(\frac{p(\tilde{x} \mid x)}{p(\tilde{x})}\right)^{q-1}\right\}-\lambda^{(1)}(x)=0
$$

The scaled Lagrange multiplier is $-\lambda^{(1)}(x)=-\lambda(x)-p^{(2-q)}(x)+\beta \sum_{y} p(y) \frac{p^{q}(x \mid y)}{p^{(q-1)}(x)}$. The Markov consistency condition $p(\tilde{x} \mid x)=\frac{p(\tilde{x} \mid y)}{p(x \mid y)}$ with Bayes rule $\frac{p(\tilde{x} \mid y)}{p(\tilde{x})}=\frac{p(y \mid \tilde{x})}{p(y)}$, yields

$$
p(\tilde{x} \mid x)=\frac{p(\tilde{x}) p(y \mid \tilde{x})}{p(y) p(x \mid y)} .
$$

Substituting (23) into (22), followed by algebraic manipulations to introduce the generalized K-Ld with the aid of (7), yields

$$
\left[\frac{q}{q-1}\left(\frac{p(\tilde{x} \mid x)}{p(\tilde{x})}\right)^{q-1}+\beta\left\{q \sum_{y} p(y \mid x) \ln _{q}\left(\frac{p(y \mid x)}{p(y \mid \tilde{x})}\right)\right\}\right]-\lambda^{(2)}(x)=0 .
$$

Here, $-\lambda^{(2)}(x)=\oplus_{q} q \beta \sum_{y} p(y \mid x) \ln _{q}\left(\frac{p(y)}{p(y \mid x)}\right)-\lambda^{(1)}(x) / p(x)+\frac{q \beta}{1-q} \sum_{y} p(y \mid x)$. Solving (24) for $p(\tilde{x} \mid x)$ yields

$$
p(\tilde{x} \mid x)=p(\tilde{x})\left[\frac{q-1}{q}\left\{-q \beta \sum_{y} p(y \mid x) \ln _{q}\left(\frac{p(y \mid x)}{p(y \mid \tilde{x})}\right)+\lambda^{(2)}(x)\right\}\right]^{1 /(q-1)} .
$$


Multiplying (24) by $p(\tilde{x} \mid x)$, and, summing over $\tilde{x}$ yields

$$
\lambda^{(2)}(x)=\frac{q}{q-1} \aleph_{q}(x)+q \beta\left\langle\sum_{y} p(y \mid x) \ln _{q}\left(\frac{p(y \mid x)}{p(y \mid \tilde{x})}\right)\right\rangle_{p(\tilde{X} \mid X=x)} .
$$

Here, $\aleph_{q}(x)=\sum_{\tilde{x}} p(\tilde{x})\left(\frac{p(\tilde{x} \mid x)}{p(\tilde{x})}\right)^{q}$. Substituting (26)into (25) yields

$$
p(\tilde{x} \mid x)=p(\tilde{x})\left\{\aleph_{q}(x)-(q-1) \beta \Delta I_{q}\right\}^{\frac{1}{(q-1)}},
$$

where, $\Delta I_{q}=\sum_{y} p(y \mid x) \ln _{q}\left(\frac{p(y \mid x)}{p(y \mid \tilde{x})}\right)-\left\langle\sum_{y} p(y \mid x) \ln _{q}\left(\frac{p(y \mid x)}{p(y \mid \tilde{x})}\right)\right\rangle_{p(\tilde{X} \mid X=x)}$. Setting $q \rightarrow$ $2-q^{*}$ and evoking (6), yields an expression in the form of the $q$-deformed exponential

$$
p(\tilde{x} \mid x)=p(\tilde{x}) \frac{\exp _{q^{*}}\left\{-\beta_{I B}^{*} \sum_{y} p(y \mid x) \ln _{q}\left(\frac{p(y \mid x)}{p(y \mid \tilde{x})}\right)\right\}}{\Im_{I B}^{\frac{1}{(1-q)}}}=p(\tilde{x}) \frac{\exp _{q^{*}}\left\{-\beta_{I B}^{*} I_{q}[p(y \mid x) \| p(y \mid \tilde{x})]\right\}}{\Im_{I B}^{\frac{1}{(1-q)}}} .
$$

In (28), the effective trade-off parameter, and, the partition function evaluated at each point in the source distribution are

$$
\begin{aligned}
& \beta_{I B}^{*}=\frac{\beta}{\Im_{I B}} \text {, and, } \tilde{Z}\left(x, \beta_{I B}^{*}\right)=\Im_{I B}^{\frac{1}{(1-q)}}, \\
& \text { where, } \Im_{I B}=\aleph_{q}(x)+(q-1) \beta\left\langle\sum_{y} p(y \mid x) \ln _{q}\left(\frac{p(y \mid x)}{p(y \mid \tilde{x})}\right)\right\rangle_{p(\tilde{X} \mid X=x)},
\end{aligned}
$$

respectively. The effective trade-off parameter for the IB method relates to the trade-off parameter as

$$
\beta=\frac{\beta_{I B}^{*} \aleph_{q}(x)}{\left[1-(q-1) \beta_{I B}^{*}\left\langle\sum_{y} p(y \mid x) \ln _{q}\left(\frac{p(y \mid x)}{p(y \mid \tilde{x})}\right)\right\rangle_{p(\tilde{X} \mid X=x)}\right]}
$$

From (28), one obtains the iterative relation

$$
p^{(m+1)}(\tilde{x} \mid x) \leftarrow \frac{p^{(m)}(\tilde{x})}{\tilde{Z}^{m+1}\left(x, \beta_{I B}^{*}\right)} \exp _{q^{*}}\left\{-\beta_{I B}^{*} I_{q}\left[p(y \mid x) \| p^{(m)}(y \mid \tilde{x})\right]\right\} .
$$

Here, (31) represents the primary distinction between the nonextensive IB method and the extensive IB method. Comparing (31) to the equivalent expression in the method IB method [8], readily reveals that in the nonextensive case the exponential term is replaced by the qdeformed exponential and the K-Ld by the generalized K-Ld. The Markov relation $\tilde{X} \leftrightarrow$ $X \leftrightarrow Y$ yields relations common to both nonextensive and extensive IB methods [18]

$$
p^{(m)}(\tilde{x})=\sum_{x} p(x) p^{(m)}(\tilde{x} \mid x), \text { and }, p^{(m)}(y \mid \tilde{x})=\frac{1}{p^{(m)}(\tilde{x})} \sum_{x} p^{(m)}(\tilde{x} \mid x) p(x, y) .
$$

The gist of the simulation is to a-priori vary $\beta_{I B}^{*} \in[0, \infty]$, followed by an a-posteriori solution of (31) and (32) in a manner akin to the EM algorithm [19]. 
Acknowledgements

This work was supported by RAND-MSR contract CSM-DI \& S-QIT-101107-2005. Gratitude is expressed to A. Plastino, S. Abe, and, K. Rose for helpful discussions.

\section{REFERENCES}

[1] C. Tsallis. Possible Generalizations of Boltzmann-Gibbs Statistics. J. Stat. Phys., 542, pp 479-487, 1988.

[2] M. Gell-Mann and C. Tsallis (Eds.). Nonextensive Entropy-Interdisciplinary Applications. Oxford University Press, New York, 2004.

[3] C. Tsallis. Generalized Entropy-Based Criterion for Consistent Testing. Phys. Rev. E, 58, pp 1442-1445, 1998.

[4] T. Cover and J. Thomas. Elements of Information Theory. John Wiley \& Sons, New York, NY, 1991.

[5] T. Berger. Rate Distortion Theory. Prentice-Hall, Englewood Cliffs, NJ, 1971.

[6] K. Rose, E. Gurewitz, and, G.C. Fox. A Deterministic Annealing Approach to Clustering. Phys. Rev. Lett., 65, pp 945948 , 1990.

[7] K. Rose. Deterministic Annealing for Clustering, Compression, Classification, Regression, and Related Optimization Problems. Proc. IEEE, 86,11, pp 22102239 , 1999.

[8] N. Tishby, F. C. Pereira, W. Bialek. The Information Bottleneck Method . Proceedings of the $37^{\text {th }}$ Annual Allerton Conference on Communication, Control and Computing, pp 368-377, 1999.

[9] P.T. Landsberg and V. Vedral. Distributions and Channel Capacities in Generalized Statistical Mechanics. Phys. Lett. A, 247, pp. 211-217, 1998.

[10] T. Yamano. Information Theory based on Nonadditive Information Content. Phys. Rev. E, 63, pp. 046105-046111, 2001.

[11] T. Yamano. Generalized Symmetric Mutual Information Applied for the Channel Capacity. Phys. Rev. E., To Appear. Manuscript available at http://arxiv.org/abs/condmat/0102322.

[12] R. E. Blahut. Computation of Channel Capacity and Rate Distortion Functions. IEEE Trans. on Inform. Theory, IT18, pp. 460473, 1972.

[13] E. Borges. A Possible Deformed Algebra and Calculus Inspired in Nonextensive Thermostatistics. Physica A, 340, pp. 95-111, 2004.

[14] E. M. F. Curado and C. Tsallis. Generalized Statistical Mechanics: Connection with Thermodynamics. Journal of Physics A, 24, pp. L69-72, 1991.

[15] C Tsallis, RS Mendes, and A. R. Plastino. The Role of Constraints Within Generalized Nonextensive Statistics Physica A, 261, pp. L534, 1998.

[16] G. L. Ferri, S. Martinez, and A. Plastino. Equivalence of the Four Versions of Tsallis Statistics. J. Stat. Phys., 04, pp. 04009-04024 , 2004. Manuscript available at http://arxiv.org/abs/cond-mat/0503441.

[17] S. Abe and A. K. Rajagopal. Nonadditive Conditional Entropy and its Significance for Local Realism. Physica A, 289,(1), pp. 157-164, 2001.

[18] N. Slonim. The Information Bottleneck: Theory and Applications. Ph.D. dissertation, Hebrew University of Jerusalem NJ, 2003. Manuscript available at http://www.princeton.edu/nslonim/.

[19] G. J. McLachlan and T. Krishnan. The EM Algorithm and Extensions. Wiley, N. Y., 1996. 